\title{
Role of Calcitonin Gene Related Peptide (CGRP) in Migraine
}

\author{
ALI M A ${ }^{1}$, RAHAMAN H²
}

\begin{abstract}
:
Migraine is the second most primary headache. The prevalence of Migraine is $12 \%$ in the general population, including $18 \%$ in women and $6 \%$ in men. Migraine can start in childhood and adolescence and continue throughout lifespan. It is most prevalent among people in their 30s and 40s. Migraine is a debilitating hemicranial headache that is pulsating, aggravated by movement, nausea, vomiting and having sensitivity to light and sound, with or without aura. It can affect all aspects of life as work and school, parenting and family relationships and personal and leisure time. There are some theory regarding pathogenesis of migraine which includes cortical spreading depression, cortical spreading oligemia, activation of trigeminocervical complex leading to neuroinflammation \& release of vasodialating neuropeptides which include calcitonin gene related peptide (CGRP), substance $P$, vasoactive intestinal polypeptide (VIP), nitric oxide (NO), and pituitary adenylate cyclase activating peptide (PACAP) \& genetic factor. CGRP is a potent vasodilator and causes perivascular plasma protein extravasation and nociceptive pain. Newer medications target CGRP both for acute and preventive treatment of migraine.
\end{abstract}

Keywords: CGRP, Gepants, mAbs.

\section{Overview:}

Migraine is a debilitating headache that is pulsating, aggravated by movement, nausea, vomiting and sensitivity to light and sound, all of these with or without aura ${ }^{1}$. A stable pattern (at least 6 months) of episodic disabling headache is usually migraine ${ }^{2}$. About $18 \%$ of adult women and $6 \%$ of men meet criteria for migraine ${ }^{3}$. Migraine can start in childhood and adolescence and continue throughout lifespan. It is most prevalent among people in their 30s and 40s, when they are busy with multiple roles and responsibilities ${ }^{4}$. It can affect all aspects of life as work and school, parenting and family relationships and personal and leisure time $^{5}$. Two and half percent of episodic migraine cases convert to chronic migraine in 1 year. The modifiable risk factors are obesity, caffeine overuse, stressful life events, sleep disorders as snoring, poor acute treatment efficacy, allodynia, anxiety and depression. The nonmodifiable risk factors are female sex, low socioeconomic status, genetics ${ }^{5}$.

\section{Diagnosing migraine:}

ICHD-3 (International Classification of Headache Disorder-3 ${ }^{\text {rd }}$ edition) Diagnostic criteria ${ }^{6}$ :
A. At least five attacks fulfilling criteria $B-D$
B. Headache attacks lasting 4-72 hours (untreated or unsuccessfully treated)
C. Headache has at least two of the following four characteristics:
1. Unilateral location
2. Pulsating quality
3. Moderate or severe pain intensity
4. Aggravation by or causing avoidance of routine physical activity (e.g. walking or climbing stairs)
D. During headache at least one of the following:

1. Nausea and/or vomiting

2. Photophobia and phonophobia

E. Not better accounted for by another ICHD-3 diagnosis.

1. Prof. Md. Ashraf Ali, Professor and Senior Consultant, Department of Neurology. Labaid Specialized Hospital, Dhaka, Bangladesh.

2. Dr. Habibur Rahaman, Phase B (MD Neurology), BSMMU, Dhaka, Bangladesh. 
Brief screeners for diagnosing migraine-

ID migraine: 3 questions examining:

Presence or absence of nausea.

Presence or absence of photophobia.

Presence or absence of impact or disability.

Single criterion diagnosis-

Patient with episodic recurrent stable headache and nausea associated with

Headaches ${ }^{3}$.

ICHD-3 (International Classification of Headache Disorder-3 ${ }^{\text {rd }}$ edition) Diagnostic Criteria for Episodic VS Chronic migraine.

Frequency determines subtypes-

Episodic migraine-Fewer than 15 headache days per month.

Chronic migraine - 15 or more headache days per month for at least 3 months.

\section{Pathophysiology of Migraine:}

There are some theory regarding pathogenesis of migraine which includes cortical spreading depression, cortical spreading oligemia, activation of trigeminocervical complex leading to neuroinflammation \& release of vasodialating neuropeptides which include calcitonin gene related peptide (CGRP), substance $P$, vasoactive intestinal polypeptide (VIP), nitric oxide (NO), and pituitary adenylate cyclase activating peptide (PACAP), genetic contribution ${ }^{7}$. Some sorts of central generator or switch which turns on pathways that go out to the meninges. In the meninges, there are peripheral pain mechanisms that actually are the source of migraine pain. What happens in meninges_- Release of neuroinflammatory and vasodilating peptides and that combination of neurogenic inflammation and vasodilation activate and sensitize nociceptive afferents that carries signal back into the brain for integration. CGRP is released from trigeminovascular afferents. It is a potent vasodilator and causes perivascular plasma protein extravasation and nociceptive pain. It also enhances transmission of pain signals in the central nervous system. CGRP levels are elevated in migraineurs. So the target for migraine treatment involve reversal or prevention of some of those mechanisms. Acute treatment could reverse the vasodilation by vasoconstriction or prevent the release of neuro inflammatory and vasodilating peptides.

There are two ways to doing now- one can activate serotonin 1B receptor to vasoconstrict and another activate serotonin 1D receptor to prevent the release of neuroinflammatory peptide or prevent the return of signal back to the brain stem. In addition, primary most potent vasodilating peptide is CGRP. Triptans prevent the release of CGRP while they vasoconstrict and reverse the vasodilation of CGRP. Newer medications target CGRP both for acute and preventive treatment of migraine 8,9 .

\section{Migraine Treatment:}

The goal of acute migraine treatment is to terminate an attack of migraine and decrease the risk of episodic migraine becoming chronic migraine. Ideally this is a one and done response, pain free within 2 hours, with minimal adverse events and a low likelihood of headache recurrence ${ }^{10}$.

First line Acute Treatments ${ }^{11}$.

Level A evidence for the following classes

Triptans

NSAIDs

Ergots

Consensus clinical recommendations are for use

Specific medications-

Triptans.

Ergots (Dihydroergotamine )

Nonspecific medications-

NSAIDs

Combination of Triptan + NSAID (SumatriptanNaproxen).

Antiemetics in management of acute migraine:

Antiemetic drugs- Metoclopramide, Prochloperazine. Available in oral and parenteral forms. Parenteral form more effective in reducing headache. Effective for nausea as well headache pain. Consider in patients who cannot tolerate or do not respond to NSAIDs/ triptans. Antiemetics may result in sedation and extra pyramidal adverse effects $^{12}$. 


\section{Current evidenced based migraine prevention treatments-}

\begin{tabular}{ll}
\hline Recommendation level based on evidence & Recommended drug classes \\
Level A, Established efficacy & $\begin{array}{l}\text { Beta blockers- propranolol, metoprolol Antiepileptic } \\
\text { drugs- divalproex sodium, sodium valproate, topiramate. }\end{array}$ \\
Level B, Probably effective & $\begin{array}{l}\text { Antidepressants- Amitriptyline, Venlafaxine.Beta } \\
\text { blockers - Atenolol, Nadolol. }\end{array}$ \\
Level C, Possibly effective & $\begin{array}{l}\text { Angiotensin receptor blockers Candesartan.Antiepileptic } \\
\text { drugs- Carbamazepine.Antihistamine -Cyproheptadine. }\end{array}$ \\
\hline
\end{tabular}

\section{Migraine prevention guideline varies by country ${ }^{13}$. \\ When to consider prevention}

Attack frequency $>1$ / week or $>2$ / months.

Significant interference with routine activities despite use of acute treatment and uncommon subtypes are present as hemiplegic migraine, migraine with prolonged aura or migrainous infarction ${ }^{14}$.

\section{Migraine prevention: Treatment principles}

Start low, go slow- titrate to avoid adverse effects. Choose treatments based on comorbidity and adverse effect profiles. Reevaluate treatment at regular intervals. Most preventive medications take at least 2 to 12 weeks before an effect is seen.

\section{Introduction to CGRP as a treatment target in migraine prevention- \\ CGRP is a specific treatment target for prevention} of migraine. mAbs are in late phase clinical studies for prevention of migraine. Small molecules gepants are in trials for migraine prevention. These are the first modern preventive treatments developed specifically for migraine ${ }^{15}$. In 1983, it was discovered that the Calcitonin gene produced Calcitonin gene related peptide ( CGRP) in neural tissue. In early 1985, a review article described the CGRP containing nerves are found in cranial blood vessels and the trigeminal ganglion. CGRP is released from trigeminovascular afferents. It is a potent vasodilator and causes perivascular plasma protein extravasation and nociceptive pain. It also enhances transmission of pain signals in the central nervous system. CGRP levels are elevated in migraineurs ${ }^{16}$.

In cluster headache there is significant increase in both VIP and CGRP level.

In chronic paroxysmal headache there is significant increase in both VIP and CGRP level. In cluster and chronic paroxysmal headache, all patients had CGRP release which normalized after treatment with Triptan ${ }^{17}$.

It is found in two major forms__ alpha and beta.

Alpha CGRP is found in the brain and nervous system. Beta CGRP is found in the rest of the body ${ }^{18}$.

\section{CGRP receptor mechanism:}

CGRP receptor complex

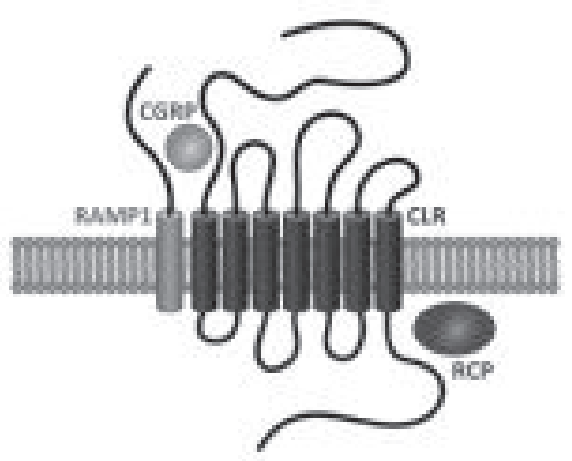

The CGRP receptor is a G-protein coupled receptor of the $B$ type. This means that it contains 1 aspect, a 7-transmembrane G protein coupled receptor-the calcitonin like receptor (CLR). To work, CLR needs an associated receptor amplifying peptide (RAMP1). When RAMP1 and CLR meet via stimulation by CGRP, the receptor is activated and the $G$ protein and the intercellular process continues ${ }^{19}$. 
CGRP receptors and migraine pathogenesisCGRP receptors are found at every area concerned with migraine pathogenesis. They are found centrally in the brain stem, in trigeminal ganglion, in the cortex, in the meninges and dura matter, around the vessels within the meninges. Activation of CGRP and release of CGRP results in meningeal vasodilation and neurogenic inflammation. That is really one of the reason why CGRP is such an attractive target for migraine prevention ${ }^{20}$.

First group of medicine that antagonizes CGRP were small molecule CGRP receptor antagonists or Gepants. They have been tested in numerous studies for acute treatment of migraine. They antagonize CGRP receptor and they terminate migraine attack. At least 6 of them have been studied in humans for acute migraine treatment. However when they are transferred to daily preventive therapy, hepatotoxicity occurred. Specifically occurred with Telcagepant, also with MK-3207. A new set of gepants has been developed for both acute and preventive treatment of episodic migraine. Two of them are Atogepant and BHV- 3500 are currently in phase 2 with daily dosing for the prevention of migraine $^{21}$.

\section{Gepants VS Triptans :}

Triptans and gepants have similar efficacy in clinical trials. Major benefit with gepants is that they do not carry cardiovascular risk. When developed, gepants are more likely to be used for triptan non responders and patients with cardiovascular disease. Gepants take longer to work and have greater tolerability ${ }^{22}$. Another approach is monoclonal antibody to CGRP or the CGRP receptor (mAbs) and they are big molecules. They do not cross blood brain barrier. They are eliminated by reticuloendothelial system. Since mAbs are not metabolized in the liver, they are not hepatotoxic. Because they do not penetrate the brain, peripheral anti CGRP action is sufficient to prevent migraine. They are working either at dura matter and meninges or in the trigeminal ganglion, both of which are external to blood brain barrier. They also have no CNS adverse effects. Four mAbs have been studied in phase 2 trials of the prevention of migraine. All four have similar very good efficacy, low rate of adverse events and very good tolerability ${ }^{23}$.

\begin{tabular}{llll}
\hline & CGRP mAbs & $\begin{array}{l}\text { Target } \\
\text { administration }\end{array}$ & Route of \\
\hline 1) & Eptinezumab & CGRP ligand & Intravenous \\
2) & Galcanezumab & CGRP ligand & SC \\
3) & Fremanezumab & CGRP ligand & SC \\
4) & Erenumab & CGRP receptor & SC \\
\hline
\end{tabular}

All mAbs look remarkably effective in preventing both episodic and chronic migraine. Sometimes patients are taking mAbs and doing very well and get a breakthrough headache. At that time Triptan may be added. Triptans may work better in the presence of a mAbs.

As CGRP is a reactive vasodilator and acts as safeguard during cerebral and cardiac ischemia. mAbs are CGRP blockers and transform mild ischemic events into full blown infarctions. So disadvantage of mAbs is that putting the susceptible patients at high risk of myocardial infarction or stroke ${ }^{24}$.

\section{Conclusion:}

CGRP provides a new target in migraine. CGRP antagonists - gepants and mAbs have the potential to really change the treatment landscape for migraine. mAbs are effective in migraine prophylaxis and gepants are effective in migraine prophylaxis and also to treat individual migraine attacks.

\section{References:}

1. Headache Classification Committee of the International Headache Society(IHS). The International classification of Headache Diorders, $3^{\text {rd }}$ edition( beta version) Cephalalgia. 2013; 33:629-808.

2. Dodic D. Diagnosing headache: clinical clues and clinical rules. Adv Stud Med. 2003; 3:5550-55.

3. Lipton RB, D Dodic, R Sadovsky et al. A self administered screener for migraine in primary care. Neurology. 2003; 61:375-82. 
4. Martin V, Donald P, Timothy $\mathrm{T}$ et al. The predictive value of abbreviated migraine diagnostic criteria. Headache. 2005; 45: 1102-12.

5. Diamon S, Bigal ME, Silberstein $S$ et al. Patterns of diagnosis and acute and preventive treatment for migraine in the United States. Headache. 2007; 47: 355-63.

6. Headache classification committee of the International Headache Society (IHS).The International classification of Headache Disorders, $3^{\text {rd }}$ edition (beta version) Cephalalgia. 2013; 33:629-808.

7. Burstein et al., 2012. Migraine: Multiple Processes, Complex Pathophysiology J Neurosci. 2015 Apr 29; 35(17): 6619-6629.

8. Goadsby PJ, Lipton RB, Ferrari MD. Migraine-current understanding and treatment. N Engl J Med. 2002; 346: 257-70.

9. Hargreaves RJ, Shepheard SL . Pathophysiology of migraine-new insights. Can J Neurol Sci. 1999; 26 suppl 3:S12-9.

10. LiptonRB, Fanning KM, Serran D et al.Ineffective acute treatment of episodic migraine is associated with new onset chronic migraine. Neurology. 2015;84:688-95.

11. Marmura MJ,Silberstein SD, Schwedt TJ. The Acute Treatment of Migraine in Adults: The American Headache Society Evidence Assessment of Migraine Pharmacotherapies. Headache. 2015;55:3-20.

12. Orr SL, Friedman BW,Christie $S$ et al.Management of adults with acute migraine in the emergency department: The American Headache Society Evidence Assessment of parenteral pharmacotherapies. Headache. 2016; 56:911-40.

13. Silberstein SD, Holland S,Freitag F.Pharmacologic treatment for episodic migraine prevention in adults. Neurology. 2012; 78:1337-45.

14. Silberstein SD.Practice parameter: evidence based guidelines foe migraine headache ( an evidence based review):report of the quality standards subcommittee of the American
Academy of Neurology. Neurology. 2000; 55:754-62.

15. Tso AR, Goadsby PJ. Anti-CGRP monoclonal Antibodies : the next era of migraine prevention? Curr Treat Options Neurol. 2017;19:27.

16. Iyengar S, Ossipov MH, Johnson KW. The role of calcitonin gene-related peptide in peripheral and central pain mechanisms including migraine. Pain. 2017;158:543-59.

17. Edvinsson L, Ho TW.CGRP Receptor Antagonism and Migraine. Neurotherapeutics. 2010;7:164-175.

18. Russel FA,King R, Smillie SJ et al.Calcitonin gene-related peptide: physiology and pathophysiology. Physiol Rev. 2014;94: 10991142.

19. Ho TW, Edvinsson L, Goadsby PJ. CGRP and its receptor provide new insights into migraine pathophysiology. Nat Rev Neurol. 2010; 6:573-82.

20. Hargreaves RJ, Shepheard SL. Pathophysiology of migraine-new insights. Can J Neurol Sci. 1999;26( Suppl 3):S12-9.

21. Diener HC,Charles A, Goadsby PJ et al.New therapeutic approaches for the prevention and treatment of migraine. Lancet Neurol. 2015;14: 1010-1022.

22. Silberstein S, Lenz R, Xu C.Therapeutic monoclonal antibodies: what headache specialists need to know. Headache. 2015; 55: 1171-82.

23. Oakes TM. CGRP monoclonal antibody for the prevention of migraine. Poster presented at AHS 2016. Abstract PS42.

24. Maassen Van de Brink A,Meijer J, Villalon CM et al. Wiping out CGRP : potential cardiovascular risks. Trends Pharmacol Sci. 2016;37: 779-88.

25. Cephalalgia. $2013 \mathrm{Jul} ; 33(9): 629-808$. The International Classification of Headache Disorders, 3rd edition (beta version). Headache Classification Committee of the International Headache Society (IHS). Comment in Cephalalgia. $2013 \mathrm{Jul} ; 33(9): 627-$ 8. Cephalalgia. 2015 Apr;35(4): 368-9. 